\title{
Strategies to Obtain Encapsulation and Controlled Release of Small Hydrophilic Molecules
}

\author{
Qi Li, Xiaosi Li and Chao Zhao* \\ Department of Chemical and Biological Engineering, The University of Alabama, Tuscaloosa, AL, United States
}

OPEN ACCESS

Edited by:

Jianxun Ding,

Changchun Institute of Applied

Chemistry (CAS), China

Reviewed by:

Jing Tang,

Stanford University, United States

Xiao Ming,

Harvard University, United States

Zhishen Ge,

University of Science and Technology

of China, China

*Correspondence:

Chao Zhao

czhao15@eng.ua.edu

Specialty section

This article was submitted to Biomaterials,

a section of the journal Frontiers in Bioengineering and Biotechnology

Received: 12 March 2020 Accepted: 16 April 2020

Published: 13 May 2020

Citation:

Li Q, Li X and Zhao C (2020) Strategies to Obtain Encapsulation and Controlled Release of Small

Hydrophilic Molecules.

Front. Bioeng. Biotechnol. 8:437.

doi: 10.3389/fbioe.2020.00437
The therapeutic effect of small hydrophilic molecules is limited by the rapid clearance from the systemic circulation or a local site of administration. The unsuitable pharmacokinetics and biodistribution can be improved by encapsulating them in drug delivery systems. However, the high-water solubility, very hydrophilic nature, and low molecular weight make it difficult to encapsulate small hydrophilic molecules in many drug delivery systems. In this mini-review, we highlight three strategies to efficiently encapsulate small hydrophilic molecules and achieve controlled release: physical encapsulation in micro/nanocapsules, physical adsorption via electronic interactions, and covalent conjugation. The principles, advantages, and disadvantages of each strategy are discussed. This review paper could be a guide for scientists, engineers, and medical doctors who want to improve the therapeutic efficacy of small hydrophilic drugs.

Keywords: small, hydrophilic, drug, encapsulation, controlled release

\section{INTRODUCTION}

Small hydrophilic molecules are widely used for treating diseases such as infectious diseases (Macielag, 2012; Zhang et al., 2015; Chandel et al., 2018), cancer (Xu et al., 2014; Zhao et al., 2016), and local anesthesia (Howell and Chauhan, 2009; Jug et al., 2010). Although effective, the dosage, therapeutic effect, and patient accomplishment of such compounds are usually limited by the tendency to distribute into the biological aqueous environment of the human body, leading to side effects (Weiniger et al., 2010; Wang et al., 2019). The pharmacokinetics and biodistribution profile of small hydrophilic molecules can be improved by encapsulating them in delivery systems which allow the sustained release and prolonging retention period. However, due to the good water solubility, hydrophilic nature, and low molecular weight, such compounds have weak interactions with many conventional drug carriers, such as hydrogels (Yu et al., 2013), poly(lactic-co-glycolic acid) microspheres (Ramazani et al., 2016), and electrospinning fibrous mat (Oliveira et al., 2015; Sultanova et al., 2016), leading to low encapsulation efficiency, undesired leakage, and initial burst release. Although many delivery systems have been attempted and shown promise in encapsulation and sustained release of hydrophilic molecules (Vrignaud et al., 2011), most of them only work well for molecules with the moderate hydrophilicity and medium molecular weight. When it comes to the super hydrophilic and very small molecules, their effectiveness is not adequate. In this review, the emphasis was given to the group of super challenging small hydrophilic molecules: compounds that have a molecular weight below $1000 \mathrm{Da}$ and have a $\log \mathrm{P}$ (partition coefficient, or XLogP3, a computed form of $\log \mathrm{P}$ ) or $\log \mathrm{D}$ (distribution coefficient) value less than 3.0 under their administration condition. In particular, tetrodotoxin 
(TTX, Mw $319.27 \mathrm{~g} / \mathrm{mol}, \log \mathrm{P}=-1.89$ ), one of the most challenging compounds to encapsulate because it is small and very hydrophilic, was selected as a reference. We introduce three efficient strategies that have been validated to encapsulate TTX and to achieve sustained TTX release, including physical encapsulation in micro/nanocapsules, physical adsorption via electronic interactions, and covalent conjugation (Figure 1). The advantages and limitations of each strategy were summarized (Table $\mathbf{1}$ ).

\section{PHYSICAL ENCAPSULATION IN MICRO/NANOCAPSULES}

Micro/nanocapsules are colloidal drug carrier systems composed of aqueous pockets surrounded by a hydrophobic membrane (Couvreur et al., 2002). Based on whether the shell is composed of lipids or polymers, the capsules are categorized as "liposomes" and "polymersomes," respectively. Liposomes (Torchilin, 2005; Chen, 2010; Eloy et al., 2014) and polymersomes (Levine et al., 2008; Anajafi and Mallik, 2015; Müller and Landfester, 2015) have been properly summarized in many other review articles. Here, we only briefly highlight the principles of encapsulating drugs in them and their associated advantages and limitations.

Liposomes and polymersomes encapsulate small hydrophilic molecules inside the internal aqueous pockets to achieve a high encapsulation efficiency. The hydrophobic shell prevents the encapsulated drug from rapid clearance, achieving sustained release (Figure 1A).

There are three types of liposomes: multilamellar vesicles, small unilamellar vesicles, and large unilamellar vesicles. The encapsulation efficiency is highly influenced by the liposome size and the drug release rate is determined by the liposome stability and shell permeability (Taylor et al., 1990; Glavas-Dodov et al., 2005). A larger internal volume leads to the higher efficiency of drug loading, while a stable liposome structure avoids the leakage of small molecular hydrophilic drugs. These essential parameters of liposomes can be adjusted to a great extent by the lipid membrane composition, chain length of the phospholipid, drug to lipid ratio, and charge property (Eloy et al., 2014).

Many liposomal formulas for small hydrophilic drugs have been FDA approved and marketed due to the high drug encapsulation efficiency, extended drug half-time, and excellent biocompatibility (Fan and Zhang, 2013). For example, DOXIL ${ }^{\circledR}$, Myocet $^{\circledR}$, and CAELYX ${ }^{\circledR}$ are marketed liposomal formulations for doxorubicin hydrochloride (DOX-HCl, Mw 580 g/mol, $\log \mathrm{D}=-0.45$ at $\mathrm{pH}$ 5.8; Dubbelboer et al., 2014), and the DAUNOXOME ${ }^{\circledR}$ is a marketed liposomal formulation for daunorubicin (Mw $527.5 \mathrm{~g} / \mathrm{mol}, \log \mathrm{P}=1.83$ ) (Chen, 2010).

Kohane and colleagues encapsulated TTX into liposomes and functionalized the liposome shell with gold nanorods (Zhan et al., 2016; Guo et al., 2020), photosensitizer (Rwei et al., 2015, 2017b), and sonosensitizer (Rwei et al., 2017a; Cullion et al., 2018), making the liposome sensitive to effects of near-infrared (NIR) light and ultrasound. The prepared formulations could release the encapsulated TTX to treat pain after operations with on-demand irradiation (Figure 2A).
The applications of liposomes are still limited by (1) instability in plasma: untailored or unmodified capsules can be adsorbed by human albumin or serum and further cleared by the immune system, inducing a short half-life in blood circulation (Wakaskar, 2018); (2) leakage during storage: the permeability of the lipid bilayer could cause leakage of the capsuled molecules during the formation process and the afterward storage (Zariwala et al., 2018), inducing an initial burst release when implemented after storage; and (3) high cost due to the expensive raw lipid materials and cumbersome production procedures.

Polymersomes have many advantages over liposomes, which give them greater potential as drug carriers. They are more stable and less permeable than liposomes due to their membrane thickness, entanglement, and lateral diffusivity (Chandrawati and Caruso, 2012; Rideau et al., 2018). Properties of polymersomes, including size, permeability, and charge property, are far more versatile than that of liposomes due to the abundantly available natural and synthetic polymer (Anajafi and Mallik, 2015; Dan, 2018; Rideau et al., 2018). The advances of polymer chemistry allow the conjugation of active ligands, functional molecules, antibodies onto the polymer, enabling polymersomes the functions of targeted and stimuli-responsive $(\mathrm{pH}$, redox, enzyme, ultrasound, magnetic field, light) drug delivery (Levine et al., 2008; Müller and Landfester, 2015; Leong et al., 2018).

However, the clinical applications of polymersomes are hampered by the residual organic solvent, incompetent control of the early drug release, cumbersome fabrication steps, and toxicity concerns (Vrignaud et al., 2011; Anajafi and Mallik, 2015).

\section{PHYSICAL ADSORPTION}

Physical adsorption strategy refers to that the molecules are physically adsorbed on carriers via inter-molecule interactions, such as ionic interaction, H-bond, van der Waals forces, hydrophobic interactions, dipole-dipole interactions (Margenau and Kestner, 2013). For the delivery of small hydrophilic molecules, ionic interaction is more preferred since others are weaker and less efficient (Yu et al., 2017) (Figure 1B).

Such a strategy is commonly utilized to load drugs into nanoparticles, silica nanoparticles, magnetic nanoparticles (Guo et al., 2020), and carbon nanodots (Tang et al., 2013). Yu utilized bioactive glass nanoparticles (BGNs) as carriers to load two different model drugs-diclofenac sodium (DS, Mw $318.13 \mathrm{~g} / \mathrm{mol}, \operatorname{LogD}=1.1$ at $\mathrm{pH} 7.4)$ and 5-fluorouracil $(5-\mathrm{Fu}$, Mw $130.08 \mathrm{~g} / \mathrm{mol}, \mathrm{XLogP} 3=-0.9$ ) (Carrer et al., 2018). GNs demonstrated $\sim 45$ folds improvement of DS loading because of the strong ionic interaction between the calcium iron of BGNs and the carboxylate group of the drug. On the contrary, BGNs showed limited loading capability to 5 -Fu because there are weak electronic interactions between BGNs and 5-Fu (Yu et al., 2017). Liu loaded the positively charged TTX into the negatively charged silica nanoparticles through electronic interactions (Figure 2B). The resulted formulation achieved sustained TTX release and decreased TTX toxicity (Liu et al., 2018).

Polyelectrolytes (PEs) are polymers that have repeating units bearing electrolyte groups. When placed in the ionizing 


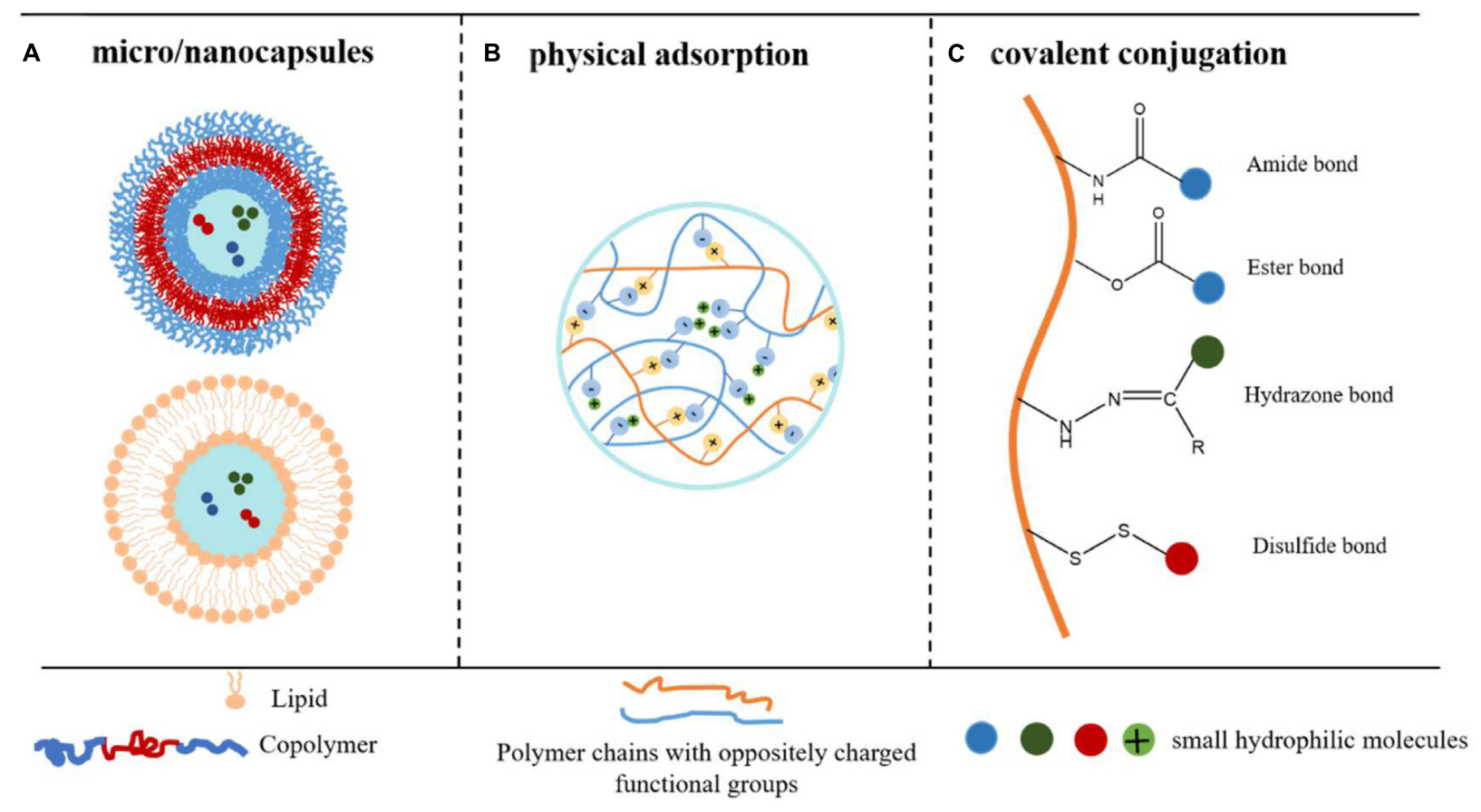

FIGURE 1 | Strategies to obtain encapsulation and controlled release of small hydrophilic molecules. (A) Drugs are physically encapsulated inside the aqueous pockets of the micro/nanocapsules. (B) Drugs are physically encapsulated in the polymer particles via electronic interaction. (C) Drugs are chemically conjugated onto polymer backbones via covalent bonds.

TABLE 1 | Advantages and limitations of strategies for small hydrophilic molecules.

\begin{tabular}{|c|c|c|}
\hline & Advantages & Limitations \\
\hline Micro/nanocapsules & $\begin{array}{l}\checkmark \text { High drug encapsulation efficiency } \\
\checkmark \text { Good biocompatibility } \\
\checkmark \text { High modifiability }\end{array}$ & $\begin{array}{l}\checkmark \text { Instability in plasma } \\
\checkmark \text { Leakage during storage } \\
\checkmark \text { High cost } \\
\checkmark \text { Toxicity related to solvents }\end{array}$ \\
\hline Physical adsorption & $\begin{array}{l}\checkmark \text { Easy operation } \\
\checkmark \text { High biocompatibility } \\
\checkmark \text { Less toxicity related to solvents and chemical crosslinking agents }\end{array}$ & $\begin{array}{l}\checkmark \text { Initial rapid drug release } \\
\checkmark \text { Less controllability }\end{array}$ \\
\hline Covalent conjugation & $\begin{array}{l}\checkmark \text { Controllable drug loading } \\
\checkmark \text { Enhanced stability } \\
\checkmark \text { Stimuli-responsive release }\end{array}$ & $\checkmark$ Toxicity related to solvents and coupling agents \\
\hline
\end{tabular}

solvent, PE will dissociate into polycations and polyanions. Then, ionized PEs in the solution can form a complex with oppositely charged PEs-a PE complex (PEC) (Meka et al., 2017). Such "chaotic" aggregation of polyanions and polycations might only be the result of partial mutual charge compensation, leaving a huge number of ionic sites compensated by small molecules with counter ions to preserve the electro neutrality (Philipp et al., 1989).

One major type of $\mathrm{PE}$ is natural polysaccharide such as chitosan, auricularia auricular polysaccharide (Xiong et al., 2016), alginate, and hyaluronic acid. They are charged due to the possession of a considerable number of charged functional groups such as carboxyl and amino groups (Liu et al., 2008), which cannot only capture and entrap hydrophilic drugs but also compact the polymer chains into stable nanoparticles.
The physical adsorption method can be considered as an energy-efficient way to achieve a high loading capacity by merely mixing the small molecules and carriers under ambient temperature. Physical adsorption is also advantageous in the diversity of polymers, which allowed the regulation of drug encapsulation efficiency, drug release profile, physical/chemical property, and biocompatibility of the PEC (Philipp et al., 1989). Besides, this strategy reduces the use of solvents and chemical crosslinking agents, addressing the potential toxicity problem (Lankalapalli and Kolapalli, 2009).

One shortcoming associated with the physical adsorption strategy is the initial rapid drug release, which is due to the saturation of the counter-ions of the carriers or the fast ion exchange (Xiong et al., 2016; Yu et al., 2017). Besides, the release profile of the drug significantly impacts on the $\mathrm{pH}$, the salt 


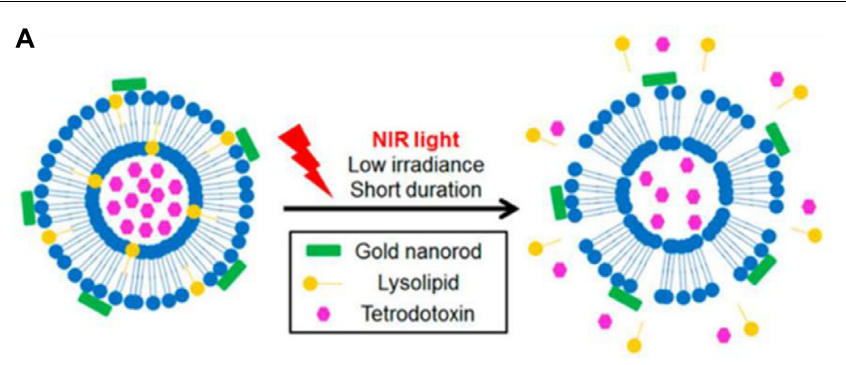

B

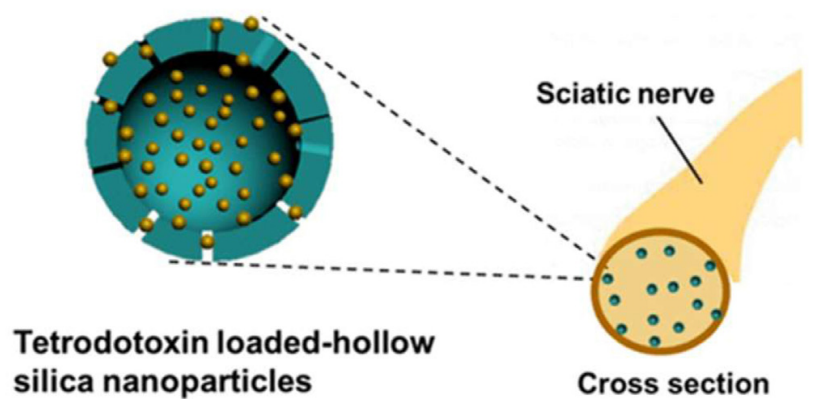

C
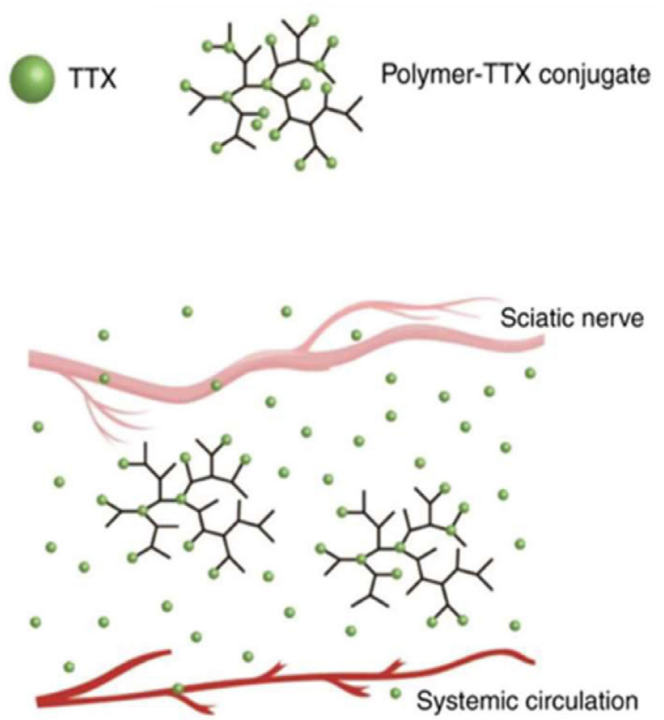

FIGURE 2 | Strategies that have been validated to encapsulate tetrodotoxin (TTX). TTX is a naturally occurring potent sodium channel blocker. Being small and super hydrophilic, TTX is selected as the gold standard for the drug delivery systems in this review. (A) Schematic of the photo-triggerable liposomes loaded with TTX (reprinted with permission from Nano Lett. 2017, 17, 2, 660-665). (B) Schematic of TTX-loaded hollow silica nanoparticles (reprinted with permission from Nano Lett. 2018, 18, 1, 32-37). (C) Schematic of the polymer-TTX conjugate, a (reprinted with permission from Nat Commun. 2019, 10, 2566).

concentration of the environment, which makes the drug release less controllable (Kulkarni et al., 2016).

\section{CONJUGATE DELIVERY SYSTEM}

In the conjugate delivery system, small hydrophilic molecules are covalently bonded onto the polymer or lipid chains through cleavable linkage, turned to be prodrugs and applied in a wider range of release routes (Figure 1C) (Adhikari et al., 2017; Irby et al., 2018; Markovic et al., 2019).

Conjugate delivery systems overcome the main drawbacks of non-covalent physical methods, unfavorable leakage and burst release, due to stable linkers between drugs and polymers (Chen et al., 2014). Zhao et al. (2019) synthesized a range of rationally designed PEGylated and non-PEGylated polymers to which the ultra-potent local anesthetic TTX was conjugated by hydrolysable ester bonds. Zhao demonstrated that TTX was released in its native form, and the release rate can be regulated by manipulating the polymer composition (the TTX release rate is proportional to the hydrophilicity of polymer backbone). In vivo, the polymer-TTX conjugate obviated TTX burst release to allow the administration of $80 \mu \mathrm{g}$ of TTX into rats, which that is 20-fold higher than the reported dose tolerance limits. The described formulation produced a sciatic nerve blockade lasting for 3 days in rats but did not cause any animal death or adverse effects (Figure 2C).

Conjugated delivery systems allow drug loading to be controlled by adjusting the drug-to-polymer ratio (Singh et al.,
2008). The amount of drug loaded depends on the number of reactive sites on the backbones. Besides, through selecting covalent bonds, stimuli-responsive drug release can be achieved (Dutta et al., 2019). For example, hydrazone bonds show strong stability under a neutral $\mathrm{pH}$ environment and sustained release in a lower $\mathrm{pH}$ environment (Tang et al., 2018). The disulfide bond is widely used as a reduction-responsive linker (Sun et al., 2018). These bonds can facilitate rapid and differential release of chemotherapeutic drugs in tumor cells to achieve the tumortargeted drug delivery (Chang et al., 2019).

Through chemical bonds, some inapplicable delivery system for small hydrophilic molecules can be preferable. Micelles have the typical structure containing a hydrophilic shell and a hydrophobic core. Based on the structure, micelles were investigated for encapsulating hydrophobic drugs because of the hydrophobicity of the inner core (Li et al., 2011; Jhaveri and Torchilin, 2014). While the hydrophobicity decreases their interactions with the hydrophilic drugs, leading to low loading capacity. The high and stable hydrophilic drug loading in micelles could be achieved by covalently linking the drug with polymer backbone (Liao et al., 2016; Danafar et al., 2017).

Coupling agents, catalysts, and solvents are used for the covalent conjugation of drug to polymers. The compound residue usually causes concern over their toxicity.

\section{CONCLUSION AND PERSPECTIVES}

Physical encapsulation in micro/nanocapsules, physical adsorption via electronic interactions, and covalent conjugation 
are the most efficient strategies to improve the therapeutic efficacy and to minimize side effects of small hydrophilic drugs. Among them, liposomal formulations have been clinically used due to the excellent lipid biocompatibility. The biocompatibility of other materials needs to be carefully examined before their clinical practice. Each strategy has its advantages and limitations. The selection of a delivery method depends on the drug property, desired drug dose, and the preferred drug release profile.

The route of administration would affect the effectiveness of the strategies in encapsulating small hydrophilic drugs. The three strategies described in this review would show good controlled release for the drugs administrated by the intramuscular, subcutaneous, intradermal injections. However, their effectiveness may be significantly reduced for oral and

\section{REFERENCES}

Adhikari, P., Pal, P., Das, A. K., Ray, S., Bhattacharjee, A., and Mazumder, B. (2017). Nano lipid-drug conjugate: an integrated review. Int. J. Pharm. 529:629. doi: 10.1016/j.ijpharm.2017.07.039

Anajafi, T., and Mallik, S. (2015). Polymersome-based drug-delivery strategies for cancer therapeutics. Therap. Deliv. 6:521. doi: 10.4155/tde.14.125

Carrer, V., Guzmán, B., Martí, M., Alonso, C., and Coderch, L. (2018). Lanolinbased synthetic membranes as percutaneous absorption models for transdermal drug delivery. Pharmaceutics 10:73. doi: 10.3390/pharmaceutics10030073

Chandel, A. K. S., Nutan, B., Raval, I. H., and Jewrajka, S. K. (2018). SelfAssembly of partially alkylated dextran- graft -poly[(2-dimethylamino)ethyl methacrylate] copolymer facilitating hydrophobic/hydrophilic drug delivery and improving conetwork hydrogel properties. Biomacromolecules 19:1142. doi: 10.1021 /acs.biomac. 8 b00015

Chandrawati, R., and Caruso, F. (2012). Biomimetic liposome- and polymersomebased multicompartmentalized assemblies. Langmuir 28:13798. doi: 10.1021/ la301958v

Chang, S., Wang, Y., Zhang, T., Pu, X., Zong, L., Zhu, H., et al. (2019). Redoxresponsive disulfide bond-bridged mPEG-PBLA prodrug micelles for enhanced paclitaxel biosafety and antitumor efficacy. Front. Oncol. 9:823. doi: 10.3389/ fonc.2019.00823

Chen, W., Zhang, J. Z., Hu, J., Guo, Q., and Yang, D. (2014). Preparation of amphiphilic copolymers for covalent loading of paclitaxel for drug delivery system. J. Polym. Sci. Part A Polym. Chem. 52, 366.

Chen, Z. G. (2010). Small-molecule delivery by nanoparticles for anticancer therapy. Trends Mol. Med. 16:594. doi: 10.1016/j.molmed.2010.08.001

Couvreur, P., Barratt, G., Fattal, E., and Vauthier, C. (2002). Nanocapsule technology: a review. Crit. Rev. ${ }^{\text {TM }}$ Therap. Drug Carrier Syst. 19, 99-134.

Cullion, K., Rwei, A. Y., and Kohane, D. S. (2018). Ultrasound-triggered liposomes for on-demand local anesthesia. Ther. Deliv. 9, 5-8. doi: 10.4155/tde-2017-0100

Dan, N. (2018). Design and Development of New Nanocarriers, eds A.M. Grumezescu. Norwich, NY: William Andrew Publishing.

Danafar, H., Rostamizadeh, K., Davaran, S., and Hamidi, M. (2017). Drugconjugated PLA-PEG-PLA copolymers: a novel approach for controlled delivery of hydrophilic drugs by micelle formation. Pharm. Dev. Technol. 22:947. doi: 10.3109/10837450.2015.1125920

Dubbelboer, I. R., Lilienberg, E., Ahnfelt, E., Sjögren, E., Axén, N., and Lennernäs, H. (2014). Treatment of intermediate stage hepatocellular carcinoma: a review of intrahepatic doxorubicin drug-delivery systems. Therap. Deliv. 5:447. doi: $10.4155 /$ tde. 14.11

Dutta, D., Ke, W., Xi, L., Yin, W., Zhou, M., and Ge, Z. (2019). Block copolymer prodrugs: synthesis, self-assembly, and applications for cancer therapy. Wiley Interdiscipl. Rev. Nanomed. Nanobiotechnol. 1585:1. doi: 10.1002/wnan.1585

Eloy, J. O., Claro, de Souza, M., Petrilli, R., Barcellos, J. P. A., Lee, R. J., et al. (2014). Liposomes as carriers of hydrophilic small molecule drugs: strategies to enhance encapsulation and delivery. Colloids Surf. B Biointerfaces 123:345. doi: 10.1016/j.colsurfb.2014.09.029 intravenous administrated drugs. The enzymatic digestion at acidic $\mathrm{pH}$ in the stomach would rapidly destroy the carrier structure and/or drug-carrier interaction, leading to the burst drug release. The long-term circulation in the blood would lead to the drug's early release before the carriers reaching to the target sites. The encapsulation of oral and intravenous administrated small hydrophilic molecules into carriers that could considerably improve the drug efficiency would be a significant need in the future.

\section{AUTHOR CONTRIBUTIONS}

\author{
QL, XL, and CZ wrote the manuscript.
}

Fan, Y., and Zhang, Q. (2013). Development of liposomal formulations: from concept to clinical investigations. Asian J. Pharm. Sci. 8:81.

Glavas-Dodov, M., Fredro-Kumbaradzi, E., Goracinova, K., Simonoska, M., Calis, S., Trajkovic-Jolevska, S., et al. (2005). The effects of lyophilization on the stability of liposomes containing 5-FU. Int. J. Pharm. 291:79. doi: 10.1016/j. ijpharm.2004.07.045

Guo, S., Yao, X., Jiang, Q., Wang, K., Zhang, Y., Peng, H., et al. (2020). Dihydroartemisinin-loaded magnetic nanoparticles for enhanced chemodynamic therapy. Front. Pharmacol. 11:226. doi: 10.3389/fphar. 2020.00226

Howell, B. A., and Chauhan, A. (2009). Bupivacaine binding to pegylated liposomes. Anesth. Analg. 109:678.

Irby, D., Du, C., Li, F., and States, U. (2018). Lipid-drug conjugate for enhancing drug delivery danielle. Mol. Pharmacol. 14:1325. doi: 10.1021/acs. molpharmaceut.6b01027

Jhaveri, A. M., and Torchilin, V. P. (2014). Multifunctional polymeric micelles for delivery of drugs and siRNA. Front. Pharmacol. 5:77. doi: 10.3389/fphar.2014. 00077

Jug, M., Maestrelli, F., Bragagni, M., and Mura, P. (2010). Preparation and solidstate characterization of bupivacaine hydrochloride cyclodextrin complexes aimed for buccal delivery. J. Pharm. Biomed. Anal. 52:9.

Kulkarni, A. D., Vanjari, Y. H., Sancheti, K. H., Patel, H. M., Belgamwar, V. S., Surana, S. J., et al. (2016). Polyelectrolyte complexes: mechanisms, critical experimental aspects, and applications. Artif. Cells Nanomed. Biotechnol. 44:1615. doi: 10.3109/21691401.2015.1129624

Lankalapalli, S., and Kolapalli, V. R. M. (2009). Polyelectrolyte complexes: a review of their applicability in drug delivery technology. Ind. J. Pharm. Sci. 71:481. doi: 10.4103/0250-474X.58165

Leong, J., Teo, J. Y., Aakalu, V. K., Yang, Y. Y., and Kong, H. (2018). Engineering polymersomes for diagnostics and therapy. Adv. Healthc. Mater. 7:1701276. doi: 10.1002/adhm.201701276

Levine, D. H., Ghoroghchian, P. P., Freudenberg, J., Zhang, G., Therien, M. J., Greene, M. I., et al. (2008). Polymersomes: a new multi-functional tool for cancer diagnosis and therapy. Methods 46:25. doi: 10.1016/j.ymeth.2008.05.006

Li, G., Liu, J., Pang, Y., Wang, R., Mao, L., Yan, D., et al. (2011). Polymeric micelles with water-insoluble drug as hydrophobic moiety for drug delivery. Biomacromolecules 12:2016. doi: 10.1021/bm200372s

Liao, C., Chen, Y., Yao, Y., Zhang, S., Gu, Z., and Yu, X. (2016). Cross-linked smallmolecule micelle-based drug delivery system: concept, synthesis, and biological evaluation. Chem. Mater. 28:7757. doi: 10.1021/acsami.8b22516

Liu, K. H., Chen, S. Y., Liu, D. M., and Liu, T. Y. (2008). Self-assembled hollow nanocapsule from amphiphatic carboxymethyl-hexanoyl chitosan as drug carrier. Macromolecules 41:6511.

Liu, Q., Santamaria, C. M., Wei, T., Zhao, C., Ji, T., Yang, T., et al. (2018). Hollow silica nanoparticles penetrate the peripheral nerve and enhance the nerve blockade from tetrodotoxin. Nano Lett. 18:32. doi: 10.1021/acs.nanolett. $7 \mathrm{~b} 02461$

Macielag, M. J. (2012). Antibiotic Discovery and Development. Berlin: Springer. 
Margenau, H., and Kestner, N. R. (2013). Theory of Intermolecular Forces: International Series of Monographs in Natural Philosophy. Amsterdam: Elsevier.

Markovic, M., Ben-Shabat, S., Keinan, S., Aponick, A., Zimmermann, E. M., and Dahan, A. (2019). Lipidic prodrug approach for improved oral drug delivery and therapy. Med. Res. Rev. 39:579. doi: 10.1002/med. 21533

Meka, V. S., Sing, M. K. G., Pichika, M. R., Nali, S. R., Kolapalli, V. R. M., and Kesharwani, P. (2017). A comprehensive review on polyelectrolyte complexes. Drug Discov. Today 22:1697. doi: 10.1016/j.drudis.2017.06.008

Müller, L. K., and Landfester, K. (2015). Natural liposomes and synthetic polymeric structures for biomedical applications. Biochem. Biophys. Res. Commun. 468:411. doi: 10.1016/j.bbrc.2015.08.088

Oliveira, M. F., Suarez, D., Rocha, J. C. B., De Carvalho Teixeira, A. V. N., Cortés, M. E., De Sousa, F. B., et al. (2015). Electrospun nanofibers of polyCD/PMAA polymers and their potential application as drug delivery system. Mater. Sci. Eng. C 54:252. doi: 10.1016/j.msec.2015.04.042

Philipp, B., Dautzenberg, H., Linow, K. J., Kötz, J., and Dawydoff, W. (1989). Polyelectrolyte complexes - recent developments and open problems. Prog. Polym. Sci. 14:91.

Ramazani, F., Chen, W., Van Nostrum, C. F., Storm, G., Kiessling, F., Lammers, T., et al. (2016). Strategies for encapsulation of small hydrophilic and amphiphilic drugs in PLGA microspheres: state-of-the-art and challenges. Int. J. Pharm. 499:358. doi: 10.1016/j.ijpharm.2016.01.020

Rideau, E., Dimova, R., Schwille, P., Wurm, F. R., and Landfester, K. (2018). Liposomes and polymersomes: a comparative review towards cell mimicking. Chem. Soc. Rev. 47:8572. doi: 10.1039/c8cs00162f

Rwei, A. Y., Lee, J.-J., Zhan, C., Liu, Q., Ok, M. T., Shankarappa, S. A., et al. (2015). Repeatable and adjustable on-demand sciatic nerve block with phototriggerable liposomes. Proc. Natl. Acad. Sci. U.S.A. 112:15719. doi: 10. 1073/pnas.1518791112

Rwei, A. Y., Paris, J. L., Wang, B., Wang, W., Axon, C. D., Vallet-Regí, M., et al. (2017a). Ultrasound-triggered local anaesthesia. Nat. Biomed. Eng. 1:644.

Rwei, A. Y., Zhan, C., Wang, B., and Kohane, D. S. (2017b). Multiply repeatable and adjustable on-demand phototriggered local anesthesia. J. Control. Release 251:68. doi: 10.1016/j.jconrel.2017.01.031

Singh, Y., Palombo, M., and Sinko, P. (2008). Recent trends in targeted anticancer prodrug and conjugate design. Curr. Med. Chem. 15:1802. doi: 10.2174/ 092986708785132997

Sultanova, Z., Kaleli, G., Kabay, G., and Mutlu, M. (2016). Controlled release of a hydrophilic drug from coaxially electrospun polycaprolactone nanofibers. Int. J. Pharm. 505:133. doi: 10.1016/j.ijpharm.2016.03.032

Sun, B., Luo, C., Yu, H., Zhang, X., Chen, Q., Yang, W., et al. (2018). Disulfide bond-driven oxidation/reduction-responsive prodrug nanoassemblies for cancer therapy. Nano Lett. 18, 3643-3650. doi: 10.1021/acs.nanolett. 8 b00737

Tang, H., Zhao, W., Yu, J., Li, Y., and Zhao, C. (2018). Recent development of $\mathrm{pH}$-responsive polymers for cancer nanomedicine. Molecules 24:4. doi: 10.3390/ molecules 24010004

Tang, J., Kong, B., Wu, H., Xu, M., Wang, Y., Wang, Y., et al. (2013). Carbon nanodots featuring efficient FRET for real-time monitoring of drug delivery and two-photon imaging. Adv. Mater. 25:6569. doi: 10.1002/adma.201303124

Taylor, K., Taylor, G., Kellaway, I., and Stevens, J. (1990). Drug entrapment and release from multilamellar and reverse-phase evaporation liposomes. Int. J. Pharm. 58:49.

Torchilin, V. P. (2005). Recent advances with liposomes as pharmaceutical carriers. Nat. Rev. Drug Discov. 4:145. doi: 10.1038/nrd1632
Vrignaud, S., Benoit, J. P., and Saulnier, P. (2011). Strategies for the nanoencapsulation of hydrophilic molecules in polymer-based nanoparticles. Biomaterials 32:8593. doi: 10.1016/j.biomaterials.2011.07.057

Wakaskar, R. R. (2018). General overview of lipid-polymer hybrid nanoparticles, dendrimers, micelles, liposomes, spongosomes and cubosomes. J. Drug Target. 26:311. doi: 10.1080/1061186X.2017.1367006

Wang, B., Wang, S., Zhang, Q., Deng, Y., Li, X., Peng, L., et al. (2019). Recent advances in polymer-based drug delivery systems for local anesthetics. Acta Biomater. 96:55. doi: 10.1016/j.actbio.2019.05.044

Weiniger, C. F., Golovanevski, M., Sokolsky-Papkov, M., and Domb, A. J. (2010). Review of prolonged local anesthetic action. Expert Opin. Drug Deliv. 7:737. doi: 10.1517/17425241003767383

Xiong, W., Li, L., Wang, Y., Yu, Y., Wang, S., Gao, Y., et al. (2016). Design and evaluation of a novel potential carrier for a hydrophilic antitumor drug: auricularia auricular polysaccharide-chitosan nanoparticles as a delivery system for doxorubicin hydrochloride. Int. J. Pharm. 511:267. doi: 10.1016/j.ijpharm. 2016.07.026

$\mathrm{Xu}$, J., Zhao, Q., Jin, Y., and Qiu, L. (2014). High loading of hydrophilic/hydrophobic doxorubicin into polyphosphazene polymersome for breast cancer therapy. Nanomed. Nanotechnol. Biol. Med. 10:349. doi: 10.1016/j.nano.2013.08.004

Yu, L., Ci, T., Zhou, S., Zeng, W., and Ding, J. (2013). The thermogelling PLGAPEG-PLGA block copolymer as a sustained release matrix of doxorubicin. Biomater. Sci. 1:411.

Yu, M., Xue, Y., Ma, P. X., Mao, C., and Lei, B. (2017). Intrinsic ultrahigh drug/miRNA loading capacity of biodegradable bioactive glass nanoparticles toward highly efficient pharmaceutical delivery. ACS Appl. Mater. Interf. 9:8460. doi: 10.1021/acsami.6b13874

Zariwala, M. G., Bendre, H., Markiv, A., Farnaud, S., Renshaw, D., Taylor, K. M. G., et al. (2018). Hydrophobically modified chitosan nanoliposomes for intestinal drug delivery. Int. J. Nanomed. 13:5837. doi: 10.2147/IJN.S166901

Zhan, C., Wang, W., McAlvin, J. B., Guo, S., Timko, B. P., Santamaria, C., et al. (2016). Phototriggered local anesthesia. Nano Lett. 16:177.

Zhang, X., Guo, R., Xu, J., Lan, Y., Jiao, Y., Zhou, C., et al. (2015). Poly(llactide)/halloysite nanotube electrospun mats as dual-drug delivery systems and their therapeutic efficacy in infected full-thickness burns. J. Biomater. Appl. 30:512. doi: 10.1177/0885328215593837

Zhao, C., Liu, A., Santamaria, C. M., Shomorony, A., Ji, T., Wei, T., et al. (2019). Polymer-tetrodotoxin conjugates to induce prolonged duration local anesthesia with minimal toxicity. Nat. Commun. 10:2566. doi: 10.1038/s41467-01910296-9

Zhao, S., Minh, L. V., Li, N., Garamus, V. M., Handge, U. A., Liu, J., et al. (2016). Doxorubicin hydrochloride-oleic acid conjugate loaded nanostructured lipid carriers for tumor specific drug release. Colloids Surf. B Biointerf. 145:95. doi: $10.1016 /$ j.colsurfb.2016.04.027

Conflict of Interest: The authors declare that the research was conducted in the absence of any commercial or financial relationships that could be construed as a potential conflict of interest.

Copyright (c) $2020 \mathrm{Li}$, Li and Zhao. This is an open-access article distributed under the terms of the Creative Commons Attribution License (CC BY). The use, distribution or reproduction in other forums is permitted, provided the original author(s) and the copyright owner(s) are credited and that the original publication in this journal is cited, in accordance with accepted academic practice. No use, distribution or reproduction is permitted which does not comply with these terms. 